\title{
Measuring the Static Modulus of Nuclear Graphite from Four-Point Flexural Strength Tests and DIC
}

\author{
J. D. Lord ${ }^{1, a}$, N. J. McCormick ${ }^{1, b}$, J. M. Urquhart ${ }^{1, c}$, G. M. Klimaytys ${ }^{1, d}$ \\ and I. J. Lingham ${ }^{2, e}$
}

\begin{abstract}
${ }^{1}$ National Physical Laboratory, Hampton Road, Teddington, Middlesex, TW110LW, UK ${ }^{2}$ National Nuclear Laboratory, Bristol Road, Stonehouse, Gloucestershire, GL10 3UT, UK

ajerry.lord@npl.co.uk,, bnick.mccormick@npl.co.uk, 'jeannie.urquhart@npl.co.uk, grant.klimaytys@npl.co.uk, eian.j.lingham@nnl.co.uk
\end{abstract}

Keywords: Digital Image Correlation, DIC, modulus, bend test, flexural strength, nuclear, graphite

\begin{abstract}
The paper describes a novel method based on Digital Image Correlation (DIC) for measuring the static modulus of active PGA graphite specimens during conventional four-point bend strength tests. DIC has been used in combination with finite element and numerical modelling to monitor the displacement fields developing in the specimen during testing, and calculate representative modulus values.

Details of the model and results are presented for two specimen geometries and a range of materials with different levels of exposure and density, tested in the perpendicular and parallel orientations. The calculated static modulus values from the DIC measurements confirm the trend between modulus, flexural strength and density. Comments and observations on the uncertainty in the measurement are also presented.
\end{abstract}

\section{Introduction}

Flexural strength, modulus and density are used as important parameters for gauging the condition of the graphite moderator in the UK nuclear reactors. Structural integrity assessments for the continued operation of the reactors are reliant upon property data obtained from material removed from the reactor cores. Currently the flexural strength and modulus are measured separately from four-point bend tests and ultrasonic dynamic Young's modulus (DYM) tests, but there are distinct advantages if this data could be obtained from a single test, and this was the purpose of this work.

Conventional contact displacement and strain measurement methods could not be used due to the nature and condition of the graphite material, so a method using DIC has been developed for the active graphite specimens. DIC is a full field non-contact strain measurement technique that was first developed in the 1980s [1,2]. It has seen significant development and uptake in recent years due to the availability of higher resolution cameras and increased computer power. DIC works by comparing images of a component or testpiece at different stages of deformation and tracking blocks of pixels to measure surface displacement and build up full-field $2 \mathrm{D}$ and $3 \mathrm{D}$ deformation vector fields and strain maps [3]. The position of the centre of the pixel blocks is determined to subpixel accuracy over the whole image using sophisticated correlation functions, from which the vector and strain components can be calculated. In this work DIC has been used in combination 
with inverse modelling to monitor the displacement field in the specimen during testing, calculating representative modulus values and stress-strain data from the tests, and offering the potential for additional supporting data for the graphite structural integrity assessment.

\section{Experimental Setup}

Initial test method development and validation was carried out at NPL, and tests on active material then carried out at the National Nuclear Laboratory (NNL), Sellafield using an identical test set up. The image acquisition system consisted of a PC and two 5-Megapixel cameras interfaced to a data acquisition unit for synchronizing the image capture with the analogue load signal from the Zwick test machine, Figure 1. The cameras were attached to the test machine baseplate at a fixed height and working distance to image the whole sample during testing, and illuminated with high intensity LED lighting. The specimen was imaged from both sides to improve the measurement statistics and capture any local variations in the structure or properties. The DIC data was calculated from each set of camera images and individual modulus values calculated, which were then averaged to present a single value of modulus for the specimen.

Most of the tests were carried out on rectangular beam-shaped specimens approximately $50 \times 8 \times 8$ $\mathrm{mm}$ in dimension, in 4 point bending with a $13.3 / 40 \mathrm{~mm}$ roller separation; but some were made on shorter beams, approximately $40 \times 8 \times 8 \mathrm{~mm}$ in dimension, in four-point bending with a 10/30 mm roller separation, as shown in Figure 2. Often with DIC, specimens are prepared by spraying a speckle paint pattern on the surface, but this was not necessary in this case as the DIC analysis was possible using the natural texture and machined surface of the graphite. The typical grain structure of the material can be seen clearly in Figure 2. In all cases the tests were carried out according to ASTM C651[4], at a constant displacement rate of $0.12 \mathrm{~mm} / \mathrm{min}$, and images captured at $2 \mathrm{~Hz}$, so each frame increment corresponds to a crosshead displacement of $1 \mu \mathrm{m}$.

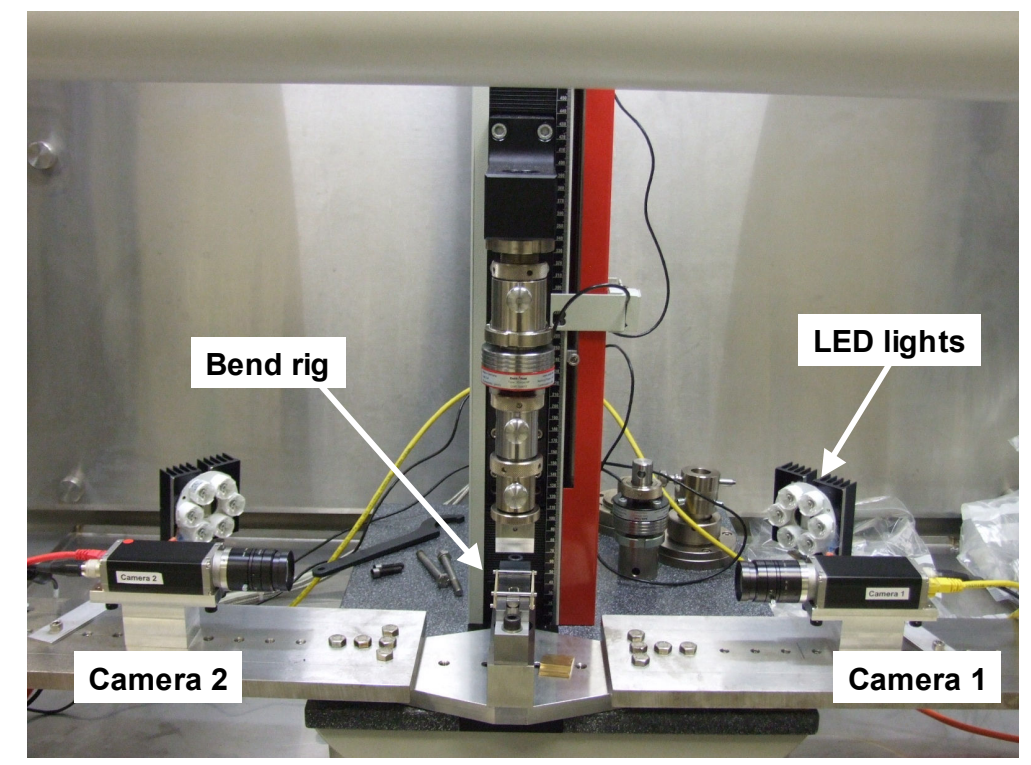

Figure 1: DIC system installed on the test machine at NNL

For each test between 100 and 200, 8-bit images were captured. Processing of the images was carried out by initial manual examination of the load-displacement data to identify the linear range over which the modulus would be calculated, followed by cropping of the images to isolate the specimen from the background and reduce the image size for calculation. 


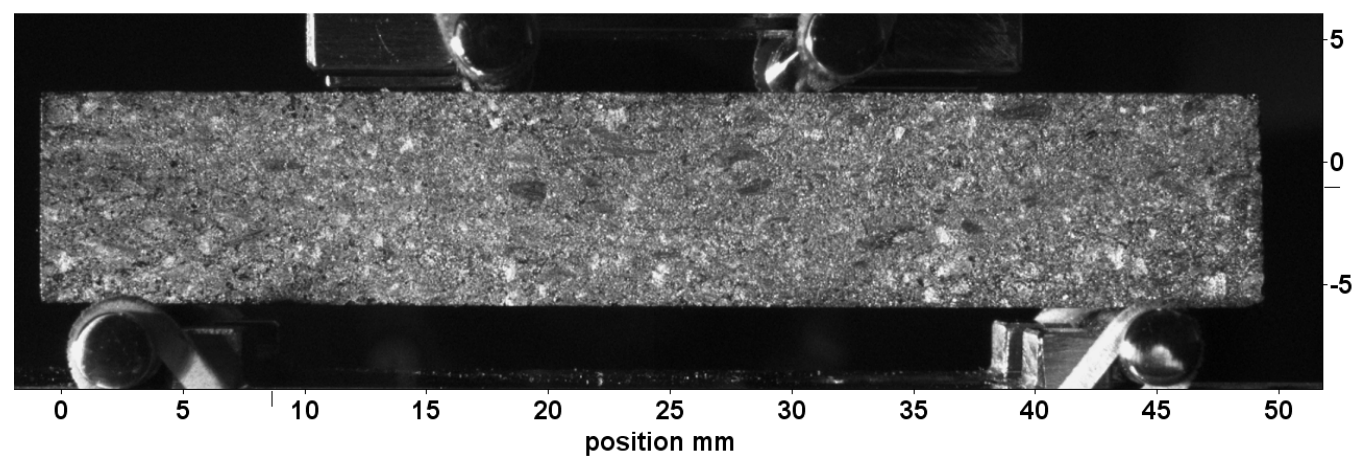

Figure 2: Close up of graphite beam

This gave a typical image size of $2100 \times 600$ pixels and a scaling of $\sim 40$ pixels $/ \mathrm{mm}$. The images were then processed using DIC to calculate the vertical displacement of the beam, Vy. The 2D DIC analysis was carried out using the LaVision Strainmaster software [5], using a "window" size of 32 x 32 pixels, with $50 \%$ overlap, giving an effective unit size of 16 x 16 pixels. This meant that approximately 2800 displacement vectors were calculated over each of the beam faces imaged. Figure 3 shows a typical load-displacement plot from the flexural test with the linear range highlighted (Frames 40-120 in this case), and the evolution of the Vy displacement field shown at different points in the test. All displacements were calculated relative to the start of the linear range - in this case selected as image 40 - but depending on the range chosen, between 4-10 profiles were generated for a particular specimen.

The displacement profiles (Vy) along the central length of the beam were then calculated and the load data and displacement profiles then input into the model for the calculation of modulus. Figure 4 shows the evolution of the beam displacement profiles during the test. The maximum deflection of the beam in the centre is $0.045 \mathrm{~mm}$.

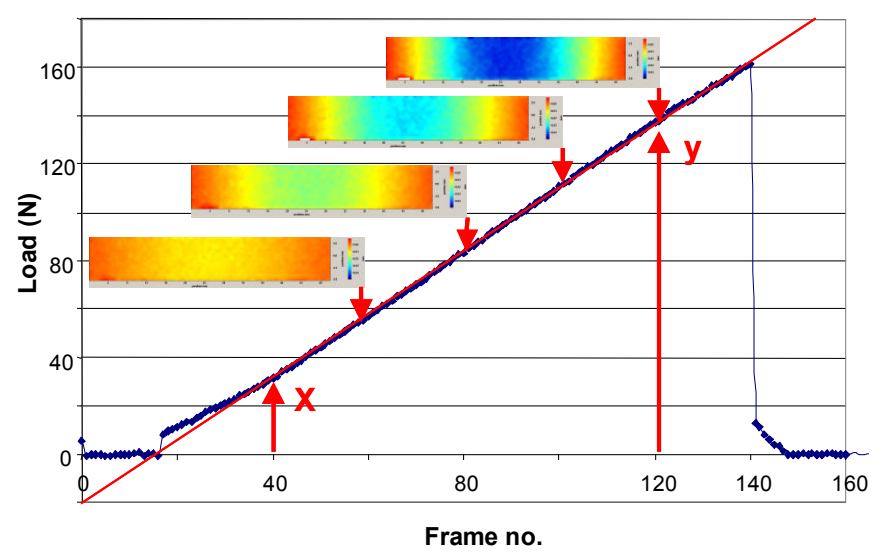

Figure 3: Typical load-displacement trace

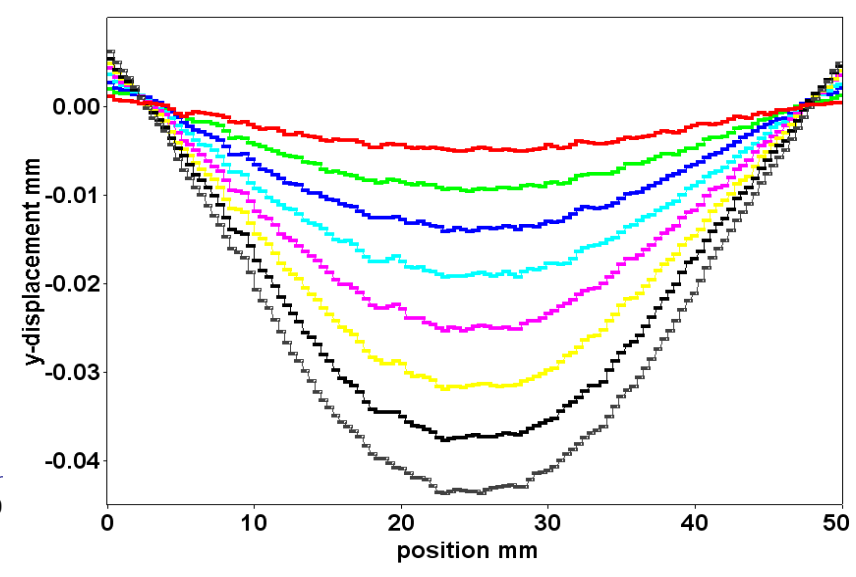

Figure 4: Corresponding beam displacement profiles

\section{Modelling}

A FEA analysis was carried out using the same sample geometry and bend rig configuration used in the test set-up, using a Young's modulus of $3 \mathrm{GPa}$ and Poisson's ratio 0.1. A 3D model was developed that was linear elastic and used 8 node cuboid elements in the Abaqus package. The size and shape of the elements chosen was not critical as only the elastic displacements in the central section of the beam were being considered, and it was not necessary to refine the mesh to model the stress concentrations under the rollers as this did not have a significant effect on the elastic behaviour and calculated centre-line displacements. Point loading for the lower rollers was achieved 
by using a line of nodes corresponding to the lower contacts, which were fixed in the vertical direction but free to move in the other directions. An area-loading model was used for contact for the top rollers. This system was used to calculate infinitesimal displacements in the vertical and horizontal directions for a plane of nodes at the mid plane of the model, assuming linear elastic behaviour. To enable an efficient analysis of the data a simple numerical model was used to fit to the FEA prediction of the $y$-displacement profile. It was found that using a function of the form:

$$
f(x)=a+b x^{2}+c x^{4}
$$

gave a good fit (Figure 5), where a, b, c are fitting parameters.

The DIC displacement profile data was first normalised by dividing the displacement data by the load increment with respect to the "reference" frame to give the unit load-deflection for the specimen. These were then averaged to improve the signal-to-noise levels, generating a single reference curve for the sample, Figure 6.

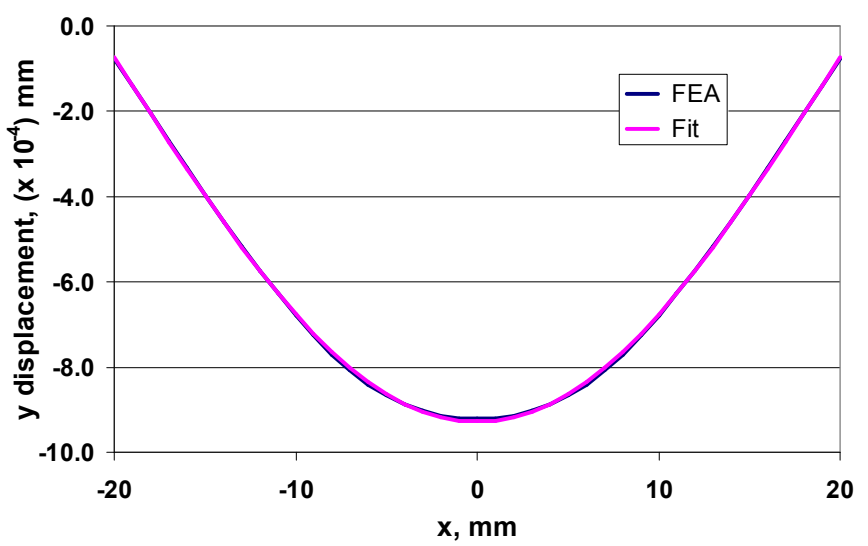

Figure 5: Comparison of FEA and analytical models

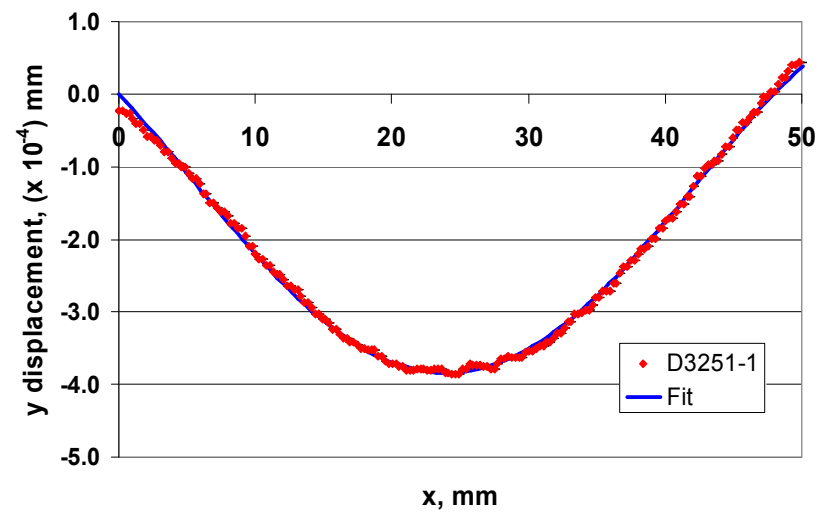

Figure 6: Normalised unit displacement profile

\section{Results}

Initial calibration tests were carried out on a series of Perspex (PMMA) specimens to check the test setup using the same test fixtures and procedures used for the graphite tests. Perspex was chosen as the "reference" material as it is homogeneous and of similar modulus to the low density irradiated material. Results were in excellent agreement with the published manufacturer's data, which gives a value for elastic modulus of $3.1 \mathrm{GPa}$.

The flexural strength data for the graphite specimens is plotted against density in Figure 7. Figure 8 shows the corresponding DIC-derived modulus data obtained from the same series of tests. In both cases the data has been normalised with respect to the properties of the virgin PGA graphite in the respective orientations. The density of the graphite beams was measured using mensuration with an assumed geometry for the volume.

Two separate batches of material, subjected to different levels of radiation damage were tested. Batch 1 samples were tested with the graphite grain structure/extrusion direction running parallel and perpendicular to the orientation of the beam; Batch 2 only in the parallel orientation. 


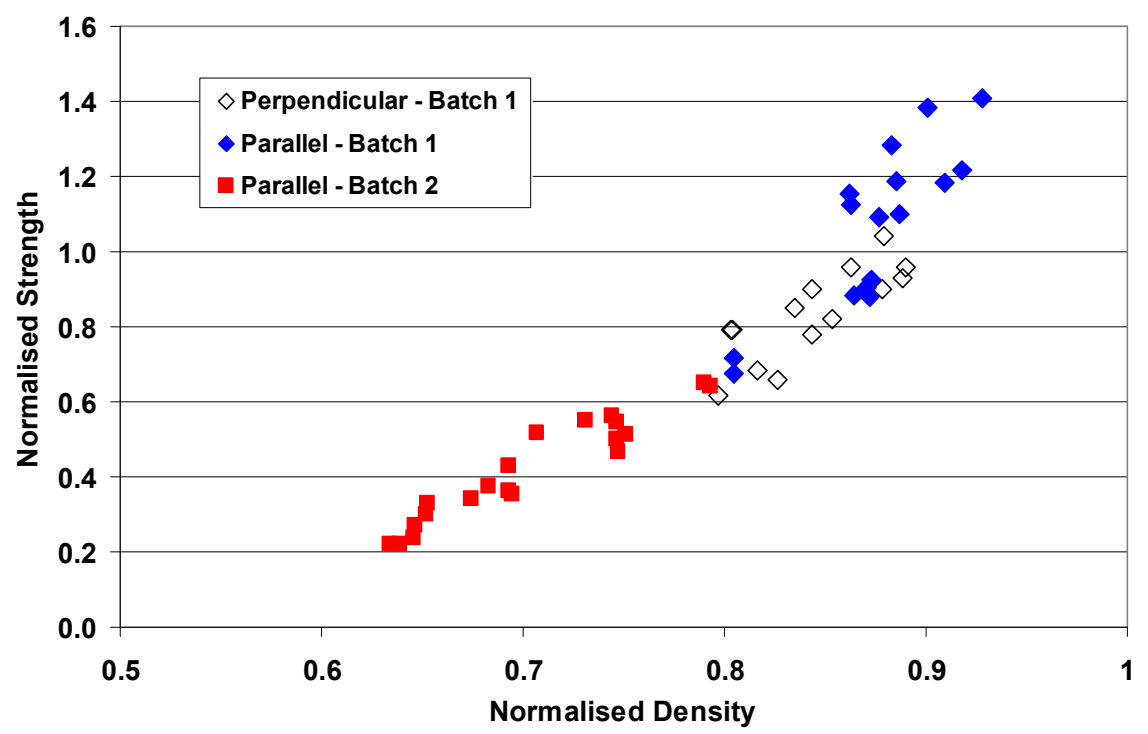

Figure 7: Flexural strength vs density

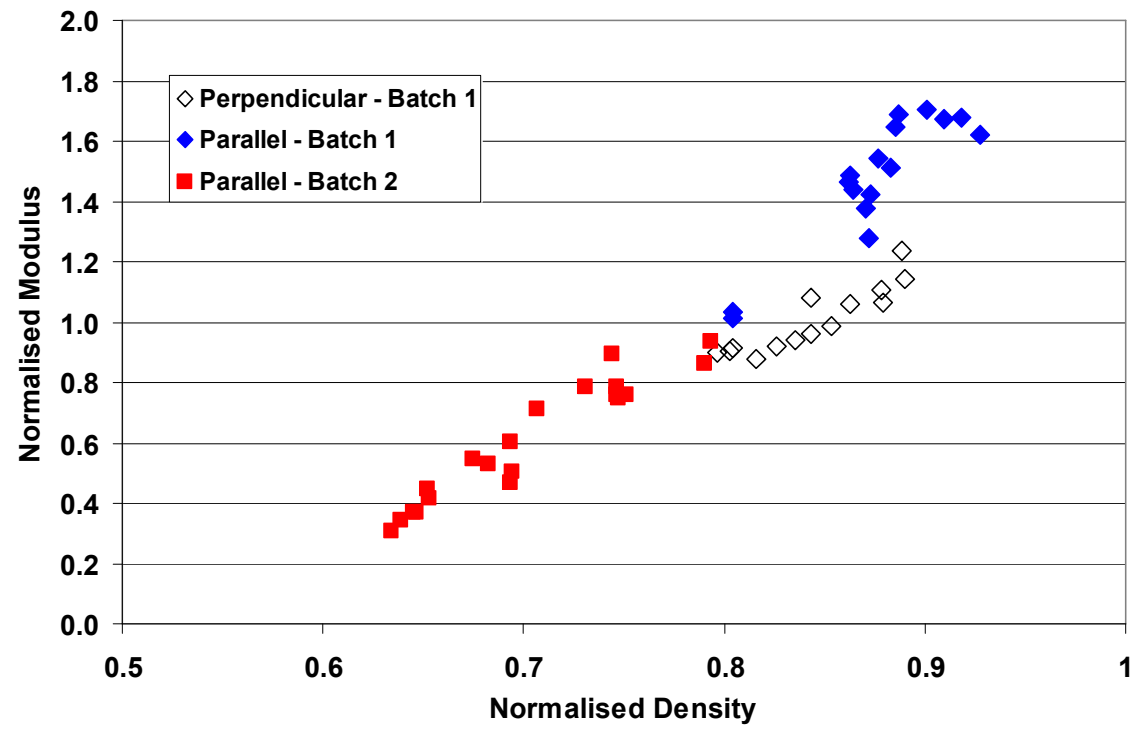

Figure 8: DIC- derived modulus data vs density

Both strength and modulus data show the same behaviour - the Batch 1 material is significantly stronger and stiffer than the Batch 2, and the material tested in the perpendicular orientation is generally less affected by the radiation (for the limited amount of samples tested). For the shorter beams from Batch 2 separate DYM measurements were made on samples cut from the end for comparison. The DIC-derived modulus data were systematically lower than the dynamic modulus values, in the ratio of $\sim 0.92$. This agrees well with the corrections used historically in the modelling and prediction of the static properties, when only dynamic modulus values were available.

The change in properties depends on the level of radiation received. In all cases there is a reduction in the density, but very different response depending on the cumulative radiation dose received. Initially the behaviour is dominated by irradiation hardening which leads to an increase in strength and modulus (Batch 1, low dose), but after further exposure radiolytic corrosion leads to weight loss and a reduction in properties (Batch 2, high dose). The two mechanisms operate simultaneously, but the effect of irradiation hardening soon saturates and the behaviour is then dominated by radiolytic corrosion. 


\section{Factors affecting the accuracy and uncertainty}

Several assumptions were made in the modelling and measurement procedure, relating to the homogeneity of the specimen, specimen shape and dimensions, perfect alignment of the rig and the positioning and alignment of the specimen within the bend rig. A pragmatic approach was taken, because although there is some anisotropy present due to specimen orientation, the structure of the graphite itself is very complex and heterogeneous, with local 3D variations in constituent materials and density, particularly with the irradiated material, which would be significantly more complex to model. At a fine scale therefore the concept of a single, constant value for modulus is bound to be insufficient, but for the purposes of measuring the representative properties of the specimen then this assumption was required.

Due to the nature of the material being tested there is potential for variations in the properties along the length of the bar. Modelling results indicate that for small variations in modulus the specimen deflections are relatively unaffected, but the shape of the curve becomes slightly asymmetric. Indeed asymmetric displacement profiles were observed in some of the tests, but further work is necessary to isolate the precise cause at this stage as there may also be contributions from misalignment of the rollers and specimen within the test jig, machining damage and dimensional variations, and local heterogeneous microstructure.

Of all the factors considered the most likely source of measurement uncertainty is probably due to specimen misalignment within the jig. There is also likely to be a contribution from material inhomogeneity and the local variations in the graphite microstructure. Based on these observations and the modelling results it is predicted that the typical uncertainty in modulus obtained from the $\mathrm{DIC} /$ flexural strength tests is of the order $1-2 \%$.

\section{Summary}

DIC has been used successfully for measuring the static modulus of active graphite samples during the four-point flexural strength test. The calculated static modulus values confirm the trend between modulus and density, and modulus and flexural strength, and the technique is able to distinguish between materials tested in different orientations. Typical uncertainties in modulus of $1-2 \%$ were achieved.

\section{References}

[1] M A Sutton and W J Wolters, Determination of displacement using an improved digital image correlation method, Image Vision Computing, 1983, 1(3), 133-139.

[2] T C Chu, W F Ranson, M A Sutton and W H Peters, Applications of digital image correlation techniques to experimental mechanics, Experimental Mechanics, 1985, 25(3), 232-244.

[3] M.A. Sutton, J-J. Orteu, and H.W.Schreier. Image Correlation for Shape, Motion and Deformation Measurements. Springer, 2009. ISBN; 978-0-387-78746-6

[4] ASTM C651 - 91(2005)e1 Standard Test Method for Flexural Strength of Manufactured Carbon and Graphite Articles Using Four-Point Loading at Room Temperature

[5] LaVision Strainmaster Davis 7.0 User Manual, November 2004

\section{Acknowledgements}

The work described was carried out as part of a contract with Magnox, who are thanked for their support and assistance. 